\title{
Semi-Supervised Online Learning of Handwritten Characters Using a Bayesian Classifier
}

\author{
Rituraj Kunwar*, Umapada Pal ${ }^{\dagger}$ and Michael Blumenstein* \\ ${ }^{*}$ School of Information and Communication Technology, Griffith University, Queensland, Australia \\ Email: rituraj.kunwar@griffithuni.edu.au, m.blumenstein@griffith.edu.au \\ ${ }^{\dagger}$ Computer Vision and Pattern Recognition Unit, Indian Statistical Institute, Kolkata, India \\ Email: umapada@isical.ac.in
}

\begin{abstract}
This paper addresses the problem of creating a handwritten character recognisor, which makes use of both labelled and unlabelled data to learn continuously over time to make the recognisor adaptable. The proposed method makes learning possible from a continuous inflow of a potentially unlimited amount of data without the requirement for storage. It highlights the use of unlabelled data for better parameter estimation, especially when labelled data is scarce and expensive unlike unlabelled data. We introduce an algorithm for learning from labelled and unlabelled samples based on the combination of novel online ensemble of the Randomized Naive Bayes classifiers and a novel incremental variant of the Expectation Maximization (EM) algorithm. We make use of a weighting factor to modulate the contribution of unlabelled data. An empirical evaluation of the proposed method on Tamil handwritten base character recognition proves efficacy of the proposed method to carry out incremental semi-supervised learning and producing accuracy comparable to state-of-the-art batch learning method. Online handwritten Tamil characters from the IWFHR 2006 competition dataset was used for evaluating the proposed method.
\end{abstract}

\section{INTRODUCTION}

In this paper we investigate the problem of automatically classifying Tamil handwritten character. Given the high popularity of tablet computers, we know there is a substantial amount of handwritten data already available and massive amounts of data are created every day. If we look at the popular classification methods in practice, then we will find numerous statistical methods which can very efficiently address this problem. There is a key restriction with most of the classification methods used currently for handwritten character recognition, which is addressed in this paper. The common constraint of most of the current classification algorithms is that they require prohibitively large amounts of labelled data to learn precisely. Labelling data is a painstaking job which involves human effort. Hence, this labelling process can be tremendously expensive and also time consuming.

Given that in most of the scripts, the number of classes to be handled is very high, thus manually labelling many samples for each class is very tedious. Therefore it would be much more preferable if a classification method required significantly less manually-labelled data instead of a large amount in order to learn and classify accurately. Another major constraint of most of the contemporary classification methods for handwritten characters is that classifiers need to be trained in an offline mode i.e. the entire training data is given in advance and the training and testing phases are separated. However if we consider a real-time problem such as handwriting recognition, where there is a continuous inflow of data and prediction is required on the fly, then in that case, offline training fails to perform adequately. If we intend to exploit the sequential inflow of data, then our learning method should have the ability to learn incrementally, where training and testing occurs in parallel. Few advantages of incremental learning are: a) very little memory requirements as it does not require the storage of training samples for future reference, b) huge amount of data can be used for better training, c) incremental methods are usually very fast, d) can handle data generated continuously with time e) can adapt if the underlying distribution of input data changes with time. In this paper we use the terms incremental learning and online learning interchangeably.

We use the proposed semi-supervised incremental variant of the EM algorithm to train (RNB) that takes advantage of both labelled and unlabelled data. EM is an iterative algorithm [1] for maximum likelihood or maximum a posteriori estimation of the incomplete data problem. Data is considered incomplete because it lacks sample class information. The algorithm first trains classifiers incrementally from scratch using the first few labelled samples and then this trained classifier is used to classify each element of unlabelled data with some posterior probability. This posterior value is used as a weight to update the learning parameters of every class. This iterative process is repeated for every unlabelled and labelled sample. In the above process, we use a weighting factor that dynamically controls the contribution of unlabelled data for the parameter estimation in EM. Next, we briefly point out the motivations and contributions of the presented work.

\section{Motivations}

(1) We use online semi-supervised learning because it is a more natural learning setting (closer to the way humans learn, leveraging from both labelled and unlabelled data).

(2) To ensure the learning system capability is adaptive to change (e.g. changes in handwriting style) in the input data. This is achieved by making the learning process online.

(3) To make the learning system capable of taking advantage of immense unlabelled data, which are cheaply available. This is achieved by making the online learning system semisupervised.

(4) The Naive Bayes (NB) is an elegant and simple classification method which in spite of being very simple, performs quite accurately for many applications (e.g. text classification). Therefore all those applications can benefit from the proposed online version of NB with slight required modifications. 
(5) The NB classifier is simple but it has much scope for further improvement by applying Bayesian network techniques.

\section{Our Contributions}

(1) To the best of our knowledge, this is the first time an online RNB has been proposed. Though it appears similar to the RNB proposed in [13], our method uses different method to update learning parameters online (eq. (4), (5) and (6)). Besides, our method uses online bagging [3] for random selection of input and they use randomized threshold selection. Moreover, unlike [13] our method takes advantage of unlabeled data.

(2) Our proposed online EM is novel. Though it is inspired by the growing GMM proposed in [2], there exist the following differences: a) the learning rate estimation step, which helps moderate the contribution of unlabelled data in the M step and hence prevents model parameters from generating improper estimates, b) estimation of variance in the $M$ step, c) the capability to handle both labelled and unlabelled data together, d) its application to a single Gaussian.

(3) To the best of our knowledge this is the first time online learning of handwritten characters has been performed in a semi-supervised setting using a Naive Bayes classifier.

Through our experimentation we prove that (1) our proposed Online RNB does converge to the offline RNB, (Fig. 1), (2) our proposed incremental semi-supervised learning method, with comparatively very few labelled samples augmented with many of unlabelled samples, does converge to the batch learning method (Fig. 2), (3) unlabelled data can significantly increase the accuracy of the classifier, especially when the available labelled data is sparse (Fig. 3) and (4) by tuning parameters of the incremental EM, we can increase or decrease the memory of the online learning system.

Handwriting recognition is among the most difficult problems of pattern recognition research, and RNB is a relatively weak classifier (as it assumes conditional independence among features) to produce an accuracy comparable to state-of-theart batch learning methods. Hence in our experiments we use fixed DTW (Dynamic Time Warping) matching in addition to semi-supervised RNB only for few confusing classes (thus minimizing the computational expense) to produce accuracy comparable to state-of-the-art classification methods. We consider a pair of classes confusing if they confuse with each other more that 10 percent of times. The DTW is considered fixed because its class templates are not updated online. DTW can be considered as a fixed post processing step.

In the following sections we present the details of our proposed methods and their performance comparisons to other contemporary methods.

\section{ONLINE RANDOM NAIVE BAYES}

\section{A. NAIVE BAYES:}

Naive Bayes [4] is well known probabilistic classifier. It assumes the conditional independence among features which greatly reduce the number of features to be estimated, and hence tremendously reduces the training time. Let us introduce some notation to describe the data. Training dataset $\chi=$ $\left(X^{1}, Y^{1}\right) \ldots\left(X^{N}, Y^{N}\right)$ where $X^{i}=x_{1}^{i} \ldots x_{D}^{i}, X^{i} \in R^{D}$ are the samples in a D dimensional feature space and $Y \in\{1 \ldots K\}$ are the corresponding labels for a K-class classification problem.
The classification rule for a new sample $X^{\text {new }}=<$ $x_{1} \ldots x_{D}>$ is:

$$
Y^{\text {new }}=\arg \max _{y_{k}} P\left(Y=y_{k}\right) \prod_{i} P\left(x_{i} \mid Y=y_{k}\right)
$$

\section{B. RANDOM NAIVE BAYES:}

RNB is an ensemble of Naive Bayes classifiers where each classifier is trained offline on different sets of training samples (Bagging) [12] and feature sets (random subspace method). For training with different sets of features we pseudo-randomly select subsets of components of the feature vector, that is, Naive Bayes is constructed on randomly chosen subspaces. To achieve this we use the Random Subspace method as proposed by T. K. Ho in [10]. Bagging and the random subspace method helps to make a diverse classifier ensemble. Classifiers made using this randomization technique are very fast (in both training and testing), easily parallelized, inherently multi-class and also stable in the sense that classifiers have a very low variance (therefore less chance of over-fitting).

Instead of using trees as classifiers with the randomization technique, we use the Naive Bayes classifier. It has been shown by Prinzie et al. [11] that other learning methods such as Naive Bayes can replace tree structures without reducing the performance. The low computational and memory cost of this classifier makes it the right choice for online learning where both computational power and memory usage is reduced.

We create each classifier in the ensemble of B classifiers by repeating the following steps B times:

1) Perform bagging to create bootstrapped training data for each classifier.

2) Randomly select $F$ features from the pool of $D$ features.

3) Estimate the learning parameters for the classifier.

A test sample will be classified by each classifier in the ensemble and the class which gets the majority vote by the ensemble will get assigned to the test sample.

\section{ONLINE RNB:}

Among one of the previous attempts to make Random Naive Bayes (RNB) [11] online was by Godec et al. [13], which uses the equally-binned histogram technique. We propose a different method to update the learning parameters in an online manner. It processes a sample only once to update the learning parameters and discard that afterwards.

We fit a univariate Gaussian $\mathcal{N}\left(x_{i} ; \widehat{\mu_{i k}}, \widehat{\sigma_{i k}^{2}}\right)$ to each $P\left(x_{i} \mid Y=y_{k}\right)$. Learning parameters (mean and variance) are updated in an online manner as shown below.

Initialization (by just one labelled training sample)

$$
\begin{gathered}
\widehat{\mu_{i k}}=x_{i k}^{1} ; \widehat{\sigma_{i k}^{2}}=\sigma_{0} . \\
\pi_{k}=\frac{1}{\text { no.ofclasses }} ; c_{k}=1 ; \alpha=\alpha_{0} .
\end{gathered}
$$

where $c_{k}=$ no. of samples used so far for training, $\alpha=$ decides the length of memory of the classifier $(\alpha<1)$ 
Repeat below steps for all the available labelled training samples:

$$
c_{k}=c_{k}+1 ; \eta_{k}=\left(\frac{1-\alpha}{c_{k}}+\alpha\right)
$$

where $\eta_{k}$ is learning rate for class $\mathrm{k}$.

$$
\begin{gathered}
\mu_{i k}(t)=\left(1-\eta_{k}\right) \mu_{i k}(t-1)+\eta_{k} x_{i}^{j} \delta\left(Y^{j}=y_{k}\right) \\
\sigma_{i k}^{2}(t)=\left(1-\eta_{k}\right) \sigma_{i k}^{2}(t-1)+\eta_{k}\left(x_{i}^{j}-\mu_{i k}(t)\right)^{2} \delta\left(Y^{j}=y_{k}\right)
\end{gathered}
$$

The classification step is the same as it is for the offline case. The Fig. 1 and the results Table 1 show that online training does converge to the offline method.

\section{ONLINE SEMI-SUPERVISED LEARNING}

In a typical case of semi-supervised learning, we have missing information in the data and the task is to estimate the value of that missing information. Usual solutions to this would be to use Expectation Maximization (EM) [1] [4]. In the Estep, the posterior probability of data points is computed, while in M-step, parameters of the learning model are computed. An iterative algorithm like this is not suitable in our context of online learning since our goal is to process training samples one at a time and to avoid their storage. This is a real-time scenario when we need to deal with streams of data or a huge dataset (which cannot be processed fully in a single step). Hence a method that updates the model in a continual and an evolutionary manner can be used in the present study. This problem has been addressed by a few researchers such as Stauffer et al. [8], Radford et al. [9], Bouchachia et al. [5] and Lee [2]. In this study we use some findings of Lee [2].

We have incorporated a regulating constant $\lambda$ in the proposed method. This parameter moderates the contribution made by the unlabelled data by reducing the learning rate $(\eta)$ and hence the weight of the unlabelled samples during step $\mathbf{M}$. The best value of $\lambda$ in practice is decided using a validation set, which was found to be 0.01 in our case. $\lambda$ reduces the learning rate for all unlabelled data equally but the value of $\eta$ increases for the correct class sample because of the higher posterior value $q_{k}$. It is a slow hill-climbing process in which the correct class sample makes the hill-climbing step greater towards the hill top, whereas the incorrect class sample makes the step small and in the opposite direction, so effectively we get closer to the hill top after processing many unlabelled data samples.

Details of the proposed incremental (online) EM algorithm for creating an incremental semi-supervised learning system are as shown below:

1) Train RNB online with a few labelled training samples. Repeat the following steps for every unlabelled or labelled data sample for better estimation of learning parameters.

2) E Step (of proposed online EM):

If incoming new sample is unlabelled then use trained classifier to find the posterior $q_{k}=P\left(Y=y_{k} \mid X\right)$ corresponding to all $\mathrm{k}$ (class). Else $q_{k}=1$.

3) M Step (of proposed online EM):

$$
c_{k}=c_{k}+q_{k} \lambda ; \eta_{k}=q_{k}\left(\frac{1-\alpha}{c_{k}}+\alpha\right) \lambda
$$

where $\lambda=$ weight factor applied to moderate the contribution of unlabelled data in the parameter estimation step ( $\lambda=1$ if the sample is Labelled) and $\eta_{k}$ is a learning rate parameter.

$$
\begin{aligned}
& \mu_{i k}(t)=\left(1-\eta_{k}\right) \mu_{i k}(t-1)+\eta_{k} x_{i}^{j} \delta\left(Y^{j}=y_{k}\right) \\
& \sigma_{i k}^{2}(t)=\left(1-\eta_{k}\right) \sigma_{i k}^{2}(t-1)+\eta_{k}\left(x_{i}^{j}-\mu_{i k}(t)\right)^{2} I
\end{aligned}
$$

where $\mathrm{I}=\delta\left(Y^{j}=y_{k}\right)$

4) Repeat step E and M (just once) for each labelled or unlabelled sample left.

The above algorithm is capable of handling both labelled and unlabelled data at the same time to update the parameters of the learning system. Experiments indicate that this online semi-supervised learning approach does converge Fig. 2 to the accuracy achieved by the offline supervised RNB with far less labelled samples. The gain in accuracy is remarkable when the labelled data used is less which is evident from Fig. 3.

\section{EXPERIMENTAL EVALUATION}

In this section we discuss Tamil handwritten character recognition, experimental settings, the database, issues with the recognition problem at hand and results. We demonstrate our proposed classification method performance on the recognition of Tamil (one of the Indian scripts) handwritten base characters. Base characters constitute only vowels and consonants. It is a 30 class problem. The IWFHR 2006 competition handwritten online dataset [18] was used for experimental results reported in this paper. Each character in the above online dataset is represented by a discrete variable number of points, and each point is described by its $\mathrm{x}$ and $\mathrm{y}$ co-ordinates.

\section{A. Experimental settings and experiments:}

For all the experiments, the data was pre-processed. For pre-processing, we normalized each character, maintaining their aspect ratio and also we resampled each character into 60 equidistant points. The five features extracted per point are a) $x$-coordinate b) $y$-coordinate c) $x$-derivative d) $y$-derivative as proposed in [6] and e) pen-direction angle as described in [7].

In all the Naive Bayes experiments we fitted a single Gaussian for each feature for each class i.e. $P\left(x_{i} \mid Y=y_{k}\right)$, where $\mathrm{i}$ and $\mathrm{k}$ represent the $i^{\text {th }}$ feature and the $k^{\text {th }}$ class respectively. For the offline RNB using the validation set (of size 100 samples), we decided upon the best number of features and classifiers in the RNB ensemble which was found to be 20 and 50 respectively. So we performed online bagging [3] to create 50 different datasets of size 350 each, chosen from a pool of 350 samples. Consequently, 50 different classifiers were created by using a set of 20 random features out of the pool of 300 and then training a classifier using a bootstrapped dataset. Classification of a test sample is performed using the majority vote. All the results reported are on a separate test set of 100 samples. Results in TABLE 1 show that RNB produced better recognition accuracy when compared to Naive Bayes. 


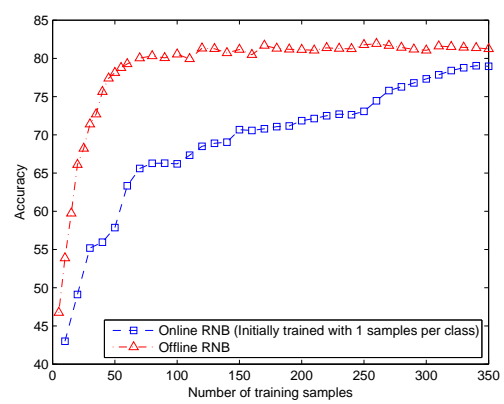

Fig. 1. Plotted accuracies of RNB and Online RNB shows that as the number of training samples increases, the gap between the accuracies of RNB and ORNB reduce monotonically. The accuracy gap increases at the beginning showing better learning by the offline RNB with few training samples.

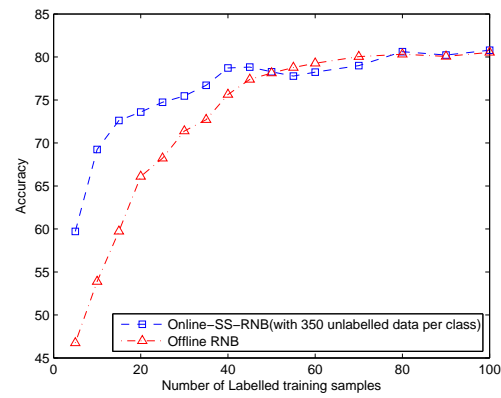

Fig. 2. Plotted accuracy of RNB and Online Semi-Supervised RNB shows that unlabelled data boosts the accuracy sharply when there is a smaller number of labelled data samples and as the number of labelled sample increases, the accuracy of online semi-supervised RNB converges to offline RNB.

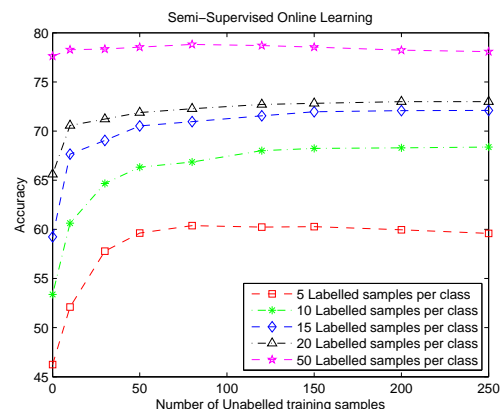

Fig. 3. Plotted accuracy shows that the Online Semi-Supervised RNB accuracy increases with unlabelled training samples. The increase in accuracy is a maximum for the classifier, which was pre-trained with a smaller number of labelled samples.

TABLE I. TABLE BELOW SHOW THE DETAIL OF EACH CLASSIFIER. 20 RANDOM FEATURES WERE USED TO CREATE 50 DIFFERENT CLASSIFIERS TO FORM THE ENSEMBLE (RNB AND ORNB). DTW-BASED

CLASSIFICATION USE 15 HAND-PICKED SAMPLES FOR EACH CLASS.

\begin{tabular}{|l|l|l|}
\hline Classifier Name & Training/Testing & Accuracy (\%) \\
\hline Naive Bayes (NB) & $350 / 100$ & 78.26 \\
\hline Random NB (RNB) & $350 / 100$ & 81.35 \\
\hline Online RNB (ORNB) & $($ Initial 2 + 350)/100 & 78.77 \\
\hline ORNB + DTW & $($ Initial 2 +350)/100 & 91.81 \\
\hline SVM & $350 / 100$ & 90.68 \\
\hline HMM & $350 / 100$ & 87.82 \\
\hline
\end{tabular}

\section{DISCUSSION}

The conditional independence assumption on which Naive Bayes is based upon is readily violated in any real world

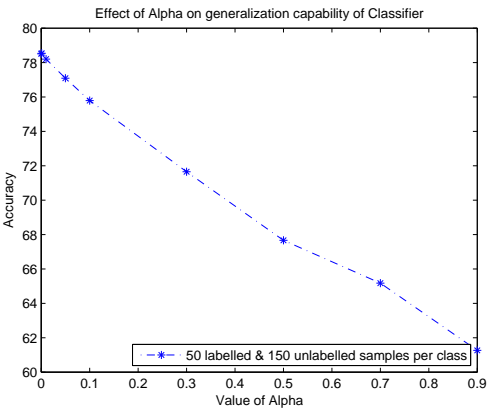

Fig. 4. Plotted accuracy shows that as the value of alpha increases (memory of classifier becomes smaller) the generalization capability of the classifier goes down.

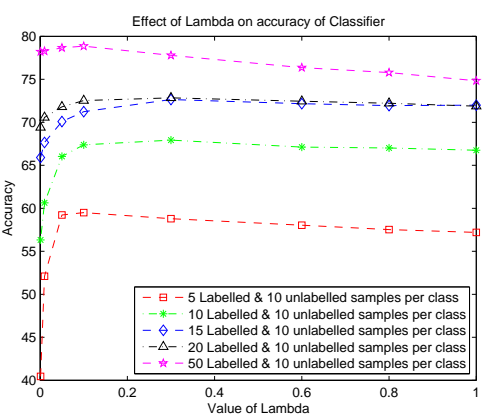

Fig. 5. Plotted accuracy shows that if number of unlabelled data is not huge then for better incremental semi supervised learning we should give relatively more weight to unlabelled data but if excessive weight is given then accuracy suffers a small dip. Number of unlabelled data used 10 per class

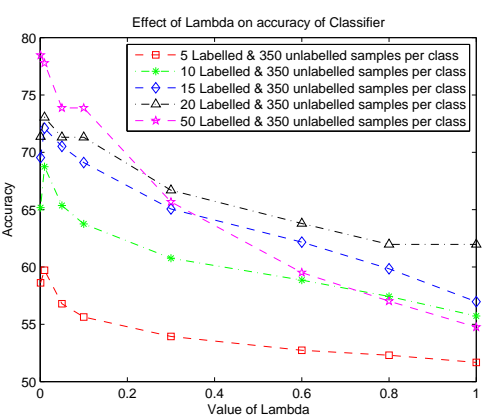

Fig. 6. Plotted accuracy shows that if number of unlabelled data is huge then for better incremental semi supervised learning we should give relatively less weight to unlabelled data otherwise accuracy suffers a huge dip. Number of unlabelled data used 350 per class

application and such is the case for handwritten character recognition. Despite that, it manages to give good accuracy as we know that a classification estimate is only a function of sign (in a 2 class problem) of function estimation. Though the estimations are poor, classification accuracy can still be high. RNB is used to improve accuracy further.

A method to learn online in a supervised manner using RNB is proposed. $c_{k}$ is one of the sufficient statistics which is updated at every step of incremental learning, and is just the count of samples from each class. $c_{k}$ is one of the parameters that decides the learning rate $\eta_{k}$. It depends on the learning rate at a particular instance of time i.e. what weight will be 
given to the new sample. Initially the $\eta_{k}$ value will be high, as the sample count would be small, thus giving more weight to the new incoming sample, but as the count grows with time, $\eta_{k}$ reduces and finally as time tends to infinity, it converges to $\alpha<<1$. This convergence to $\alpha$ makes sure that learning does not stop ever thus making the incremental supervised learning scheme adaptable. With this understanding it is possible to make an application (based on this learning scheme) where the user has the liberty to change this single parameter to increase or decrease the learning rate. For example, let us consider a device that has a handwriting recognition system and a user wants to customize that according to his handwriting style really fast, then the user has to just decrease the value of a single parameter $c_{k}$ to increase the learning rate so that it adapts faster by giving more weight to the current user's handwriting style.

Along a similar line, the proposed incremental EM makes incremental semi-supervised learning possible. Unlike the usual EM algorithm (iterative), incremental EM is executed only once for each labelled or unlabelled sample. This makes the procedure very fast. It is well understood from the literature that if our statistical model is close to the data generating model then the EM algorithm can help to extract information from the unlabelled data and improve the estimates of the parameters, else it can degrade the estimates. In the present case, since the assumption of independence among features does not hold in handwritten characters, it degrades the estimates and hence the accuracy. This is evident from Fig. 6 where it is noted that as we increase the weight of an unlabelled data contribution (i.e. as the value of $\lambda$ increases) the accuracy goes down. This phenomenon is less severe in cases when we use less unlabelled data as shown in Fig. 5. From Fig.5 and Fig. 6 we can easily ascertain that if we have more unlabelled data we should give less weight to the unlabelled data for a better estimate (in our case $\lambda=0.01$ ) as per Fig. 6. And in case where we have less unlabelled data then we should give more weight to the unlabelled data (best accuracy is achieved around $\lambda=0.3$ ), as in Fig. 5. Eventually we observed that with the increase in the number of unlabelled data, estimates of the parameters improved, which manifested in terms of higher accuracy. Thus it is evident from Fig. 6 that if parameter $\lambda$ had not been used $(\lambda=1)$ then the accuracy would have suffered a very sharp dip. As expected, the degradation in accuracy is sharper when the number of labelled data used is high.

Another crucial parameter used in incremental EM is $\alpha<<$ 1 , which can be thought of as the one which decides length of the memory of the learner. If the value of $\alpha$ is large then (learning rate $\eta$ increases) the learner has a smaller memory and gives more weight to the most recently encountered data and forgets quickly. Thus it is can be easily understood that $\alpha$ decides the generalization capacity of the learner. Fig. 4 shows that as the value of $\alpha$ increases, the memory of the learner decreases and hence the generalization capacity (classification accuracy) decreases sharply. Finally, if the learning occurs for a long time, then the learning rate value converges to $\alpha$ thus making sure that the learning does not stop ever and adapts to the changing nature of the input data. Analysis on the learning rate has been extensively conducted in [2], which precisely says that it is better for the learning rate to converge (asymptotically) to a small value $(\alpha=0.001)$ than to 0 (as proposed in [14] and [15]). And it is also not preferable to keep the learning rate constant to a small value, as used in the formulation of [16] and [17] because it takes longer for the parameters to converge.

\section{CONCLUSION}

The goal of the present work was to make a Semisupervised incremental version of the Naive Bayes classifier which should be as accurate as its supervised offline counterpart. This was successfully demonstrated on Tamil Handwritten character recognition using a standard dataset. Although it did not produce the best accuracy on its own, it did produce accuracy comparable to the state-of-the-art classifiers such as SVMs and HMM when boosted with a $2^{\text {nd }}$ stage classification method based on DTW matching. For future research we have plans to improve Naive Bayes classifier to increase its accuracy.

\section{REFERENCES}

[1] A.P. Dempster, N.M. Laird, and D.B. Rubin, Maximum Likelihood from Incomplete Data Via the EM Algorithm, J. Royal Statistical Soc. B, vol. 39, pp. 1-38, 1977.

[2] D.S. Lee Effective Gaussian mixture learning for video background subtraction, IEEE Trans. Pattern Anal. Mach. Intell., vol. 27, no. 5, pp.827 -832 2005

[3] N. C. Oza Online bagging and boosting, Proc. IEEE Int. Conf. Syst. Man Cybern., vol. 3, pp.2340 -2345 2006

[4] Kamal Nigam, Andrew McCallum, Sebastian Thrun and Tom Mitchell. Text Classification from Labeled and Unlabeled Documents using EM. Machine Learning, 39(2/3). pp. 103-134. 2000.

[5] Bouchachia, A., Prossegger, M., Duman, H. Semi-supervised incremental learning. IEEE International Conference on Fuzzy Systems (FUZZ), Pages 1-6, 2010.

[6] E. Keogh and M. Pazzani, Derivative dynamic time warping, First SIAM International Conference on Data Mining (SDM), Chicago, USA, 2001.

[7] K. Shashikiran, K. S. Prasad, Rituraj Kunwar, A. G. Ramakrishnan, Comparision of HMM and SDTW for Tamil handwritten character recognition. SPCOM 2010, pp. 1-4.

[8] C. Stauffer and W. Grimson. Learning patterns of activity using realtime tracking. IEEE Trans. Pattern Anal. Mach. Intell., 22(8):747-757, 2000.

[9] N. Radford and G. Hinton. Learning in graphical models, chapter A view of the EM algorithm that justifies incremental, sparse, and other variants, pages 355-368. MIT Press, 1999.

[10] T. K. Ho. The random subspace method for constructing decision forests. IEEE Transactions on Pattern Analysis and Machine Intelligence, 20(8):832-844, 1998.

[11] A. Prinzie and D. Van den Poel, Random multiclass classification: Generalizing random forests to random $\mathrm{mnl}$ and random $\mathrm{nb}$, in Database and Expert Systems Applications, 2007.

[12] L. Breiman, Random forests, Machine Learning, 2001.

[13] Martin Godec, Christian Leistner, Amir Saffari, Horst Bischof, On-line Random Naive Bayes for Tracking, ICPR 2010.

[14] N. Friedman and S. Russell, Image Segmentation in Video Sequences: A Probabilistic Approach, Proc. 13th Conf. Uncertainty in Artificial Intelligence, Aug. 1997

[15] M.A. Sato and S. Ishii, Online EM Algorithm for the Normalized Gaussian Network, Neural Computation, vol. 12, pp. 407-432, 1999.

[16] M. Harville, G. Gordon, and J. Woodfill, Foreground Segmentation Using Adaptive Mixture Models in Color and Depth, Proc. ICCV Workshop Detection and Recognition of Events in Video, July 2001.

[17] C. Stauffer and W.E.L. Grimson, Adaptive Background Mixture Models for Real-Time Tracking, Proc. Conf. Computer Vision and Pattern Recognition, vol. 2, pp. 246-252, June 1999.

[18] http://www.hpl.hp.com/india/research/penhw-interfaces1linguistics.html 